\title{
Modes Control of Lamb Wave in Plates Using Meander-Line Electromagnetic Acoustic Transducers
}

\author{
Yinghong Zhang, Zhenghua Qian * and Bin Wang
}

State Key Laboratory of Mechanics and Control of Mechanical Structures, College of Aerospace Engineering, Nanjing University of Aeronautics and Astronautics, Nanjing 210016, China; zyh1433@sina.com (Y.Z.); wangbin1982@nuaa.edu.cn (B.W.)

* Correspondence: qianzh@nuaa.edu.cn; Tel.: +86-25-84892696

Received: 9 April 2020; Accepted: 11 May 2020; Published: 18 May 2020

\begin{abstract}
The multimode and dispersion characteristics of Lamb waves make them difficult to apply to nondestructive evaluation. This paper presents a paired configuration of a meander-line coil electromagnetic acoustic transducer (EMAT) to generate a single-mode symmetric and antisymmetric Lamb wave in aluminum plates. In the paired structure, the bias magnetic field of the EMAT that generates symmetric mode Lamb waves is perpendicular to the plate surface, while the bias magnetic field of the EMAT that generates antisymmetric Lamb waves is parallel to the plate surface. The symmetric and antisymmetric exciting forces generated by these two EMATs are consistent with the dispersion equations of single symmetric and antisymmetric Lamb wave modes, respectively. The numerical simulations and experiments verified that the presented paired configurations of meander-line coil EMATs can effectively control the generation of single-mode Lamb waves at low frequencies.
\end{abstract}

Keywords: Lamb wave; electromagnetic acoustic transducers (EMATs); mode control; meander-line coil

\section{Introduction}

Metallic plates are widely used in aircraft, rockets, and petrochemical industries as well as in pressure vessels [1,2]. Finding cracks, pores, and slags in plates before they are processed into products is vital to avoid catastrophic failure and economic losses. Guided Lamb waves with their long-range propagation characteristics are ideally suited for inspection of plate-like structure [3-6]. In nondestructive testing, a single Lamb wave mode is very advantageous for simplifying analysis and using the combination of specific mode characteristics and frequencies. However, guided waves propagating in a plate are inherently dispersive and present an infinite number of modes. Multiple modes with different propagation velocities make it difficult to extract meaningful information from received signals.

There are some significant studies on the selective generation of single-mode Lamb waves. Single-mode Lamb waves generated by piezoelectric lead zirconate titanate (PZT) transducers or polyvinylidene fluoride (PVDF) have been demonstrated [7-10]. These methods require the transducer to be bonded to the structure and are therefore suitable for structural health monitoring but are difficult to apply when detecting moving objects or scanning. Since electromagnetic acoustic transducers (EMATs) can realize non-contact measurement in the nondestructive testing of conductive materials, they have been extensively studied. In the process of EMATs exciting guided waves, the magnitude and direction of the excitation force have a great influence on the guided wave mode, while the structure of the magnet and coil determines the magnitude and direction of the excitation force. Therefore, a common method of exciting single-mode Lamb waves is optimizing the magnet and coil structure of 
EMATs. For example, the genetic algorithm is used to optimize the diameter and the lift-off of magnet to obtain the preferred A0 mode [11], the wavelength filtering function of the double spiral coils are used to obtain the preferred S0 mode [12], and the dual magnets are used to provide the horizontal magnetic field to obtain the preferred A0 mode [13,14]. In general, according to the direction of the bias magnetic field, EMATs can be grouped into two types: Normal biased EMATs (NB-EMATs) and parallel biased EMATs (PB-EMATs). An NB-EMAT refers to the EMAT whose bias magnetic field is normal to the specimen surface [11,12], while a PB-EMAT refers to the EMAT whose bias magnetic field is parallel to the specimen surface $[13,14]$. Although the Lamb wave mode control methods proposed in [11-14] can generate a single omnidirectional Lamb wave mode, these methods are difficult to apply to the meander-line coil EMAT. Compared with the omnidirectional EMATs, the meander-line coil EMATs have higher directivity and lower amplitude attenuation, so they are more widely used in practical applications of large-area scan detection. Consequently, in this paper, we focus on the design of EMATs with meander-line coil for Lamb wave mode excitation.

At first, from the frequency equations of Lamb waves, we deduce the force conditions for the excitation of single-mode Lamb waves. After that, based on the exciting force conditions of symmetric and antisymmetric Lamb wave modes, two novel paired EMAT configurations were proposed to control the Lamb wave modes. The paired NB-EMAT configuration is used to generate symmetric modes and the paired PB-EMAT configuration is used to generate antisymmetric modes, respectively. Then we present the simulation and experimental verification; the simulations and experiments are carried out on the aluminum plate, in which the NB-EMAT works at frequency $328 \mathrm{kHz}$, and the PB-EMAT works at $366.7 \mathrm{kHz}$. Finally, we summarize the performance of the proposed paired EMAT configuration for controlling the excitation of Lamb wave modes.

\section{Lamb Waves}

It is known that the ultrasonic Lamb wave is a type of wave that remains guided between two parallel free boundaries. Lamb wave theory is fully documented in many textbooks [15-17]. Here, we only introduce the bare essentials.

The acoustic field equation can be stated in terms of a particle displacement $\mathbf{u}$ as follows:

$$
(\lambda+\mu) \nabla(\nabla \cdot \mathbf{u})+\mu \nabla^{2} \mathbf{u}=\rho \frac{\partial^{2} \mathbf{u}}{\partial t^{2}}
$$

According to the Helmholtz decomposition principle, $\mathbf{u}=\nabla \varphi+\nabla \times \psi$, the decomposition is then substituted into the field equation, and we obtain the two separated wave equations:

$$
\begin{aligned}
& \frac{\partial^{2} \phi}{\partial x^{2}}+\frac{\partial^{2} \phi}{\partial y^{2}}=\frac{1}{c_{L}^{2}} \frac{\partial^{2} \phi}{\partial t^{2}} \\
& \frac{\partial^{2} \psi}{\partial x^{2}}+\frac{\partial^{2} \psi}{\partial y^{2}}=\frac{1}{c_{T}^{2}} \frac{\partial^{2} \psi}{\partial t^{2}}
\end{aligned}
$$

where $\varphi$ and $\psi$ are two potential functions, $C_{L}^{2}=(\lambda+2 \mu) / \rho$ and $C_{T}^{2}=\mu / \rho$ are the longitudinal and transverse wave speeds, $\lambda$ and $\mu$ are the Lame constants, and $\rho$ is the mass density of the material. Based on the plane strain assumption, we assume that the solutions of Equation (2) are

$$
\begin{aligned}
\phi & =\Phi(z) \exp [i(k x-\omega t)] \\
\psi & =\Psi(z) \exp [i(k x-\omega t)]
\end{aligned}
$$

where $k$ is the wavenumber. The time dependence is assumed harmonic in the form $e^{-i \omega t}$. Substituting Equation (3) into Equation (2), the general solution of Equation (2) is

$$
\begin{aligned}
& \Phi(z)=A_{1} \sin (p y)+A_{2} \cos (p y) \\
& \Psi(z)=B_{1} \sin (q y)+B_{2} \cos (q y)
\end{aligned}
$$


and

$$
\begin{aligned}
& p^{2}=\frac{\omega^{2}}{c_{L}^{2}}-k^{2} \\
& q^{2}=\frac{\omega^{2}}{c_{T}^{2}}-k^{2}
\end{aligned}
$$

The four integration constants $\left(A_{1}, A_{2}, B_{1}\right.$, and $\left.B_{2}\right)$ are to be found from the boundary conditions. Since the exponential appears in all of the expressions, it does not play a further role in the determination of the frequency equation, and it is therefore omitted in the sequel. Using the relations between the potential functions and the displacements and stresses:

$$
\begin{aligned}
& u_{x}=\frac{\partial \phi}{\partial x}+\frac{\partial \psi}{\partial y} \\
& u_{y}=\frac{\partial \phi}{\partial y}-\frac{\partial \psi}{\partial x} \\
& \tau_{y x}=\mu\left(2 \frac{\partial^{2} \phi}{\partial x \partial y}-\frac{\partial^{2} \psi}{\partial x^{2}}+\frac{\partial^{2} \psi}{\partial y^{2}}\right) \\
& \tau_{y y}=\lambda\left(\frac{\partial^{2} \phi}{\partial x^{2}}+\frac{\partial^{2} \phi}{\partial y^{2}}\right)+2 \mu\left(2 \frac{\partial^{2} \phi}{\partial y^{2}}-\frac{\partial^{2} \psi}{\partial x \partial y}\right)
\end{aligned}
$$

and substituting Equations (3)-(5) into Equation (6), the resulting equations are obtained as

$$
\begin{gathered}
u_{x}=\left[i k A_{2} \cos (p y)+q B_{1} \cos (q y)\right]+\left[i k A_{1} \sin (p y)-q B_{2} \sin (q y)\right] \\
u_{y}=-\left[p A_{2} \sin (p y)+i k B_{1} \sin (q y)\right]+\left[A_{1} p \cos (p y)-i k B_{2} \cos (q y)\right] \\
\tau_{y x}=\mu\left[-2 i k p A_{2} \sin (p y)+\left(k^{2}-q^{2}\right) B_{1} \sin (q y)\right]+\mu\left[2 i k p A_{1} \cos (p y)+\left(k^{2}-q^{2}\right) B_{2} \cos (q y)\right] \\
\tau_{y y}=\mu\left[\left(k^{2}-q^{2}\right) A_{2} \cos (p y)-2 i k q B_{1} \cos (q y)\right]+\mu\left[\left(k^{2}-q^{2}\right) A_{1} \sin (p y)+2 i k q B_{2} \sin (q y)\right]
\end{gathered}
$$

where $u_{x}$ is the in-plane displacement component, $u_{y}$ is the out-plane displacement component, $\tau_{y y}$ is the normal stress component, and $\tau_{y x}$ is the shear stress component. The terms in Equation (10) have been split up into two systems of symmetric and antisymmetric modes, respectively:

Symmetric modes:

$$
\begin{gathered}
u_{x}=\left[i k A_{2} \cos (p y)+q B_{1} \cos (q y)\right] \\
u_{y}=-\left[p A_{2} \sin (p y)+i k B_{1} \sin (q y)\right] \\
\tau_{y x}=\mu\left[-2 i k p A_{2} \sin (p y)+\left(k^{2}-q^{2}\right) B_{1} \sin (q y)\right] \\
\tau_{y y}=\mu\left[\left(k^{2}-q^{2}\right) A_{2} \cos (p y)-2 i k q B_{1} \cos (q y)\right]
\end{gathered}
$$

Antisymmetric modes:

$$
\begin{gathered}
u_{x}=\left[i k A_{1} \sin (p y)-q B_{2} \sin (q y)\right] \\
u_{y}=\left[A_{1} p \cos (p y)-i k B_{2} \cos (q y)\right] \\
\tau_{y x}=\mu\left[2 i k p A_{1} \cos (p y)+\left(k^{2}-q^{2}\right) B_{2} \cos (q y)\right] \\
\tau_{y y}=\mu\left[\left(k^{2}-q^{2}\right) A_{1} \sin (p y)+2 i k q B_{2} \sin (q y)\right]
\end{gathered}
$$

For free wave motion, we derive the homogenous solution by applying the stress-free boundary conditions at the upper and lower surfaces at $y= \pm d$ :

$$
\left.\tau_{y x}\right|_{y= \pm d}=\left.\tau_{y y}\right|_{y= \pm d}=0
$$

where $d$ is the half-thickness of the plate.

For the symmetric modes, the boundary conditions yield a system of two homogeneous equations for the constants $A_{2}$ and $B_{1}$. Similarly, for the antisymmetric modes, two homogeneous equations for the constants $A_{1}$ and $B_{2}$ are obtained. Since the systems are homogeneous, the determinant of the 
coefficients must vanish, which yields the frequency equation. Thus, we can obtain the well-known Rayleigh-Lamb frequency equations:

$$
\begin{aligned}
& \frac{\tan (q d)}{\tan (p d)}=-\frac{4 k^{2} p q}{\left(p^{2}-k^{2}\right)^{2}} \\
& \frac{\tan (q d)}{\tan (p d)}=-\frac{\left(q^{2}-k^{2}\right)^{2}}{4 k^{2} p q}
\end{aligned}
$$

Equation (20) corresponds to symmetric (S) modes and Equation (21) to the antisymmetric (A) modes.

By reviewing the derivation process of the Lamb wave dispersion equations, we can see that if one wants to obtain a pure single-mode guided wave, the excitation force must satisfy the single-mode guided wave dispersion equation. When designing the transducer, it is necessary to consider the excitation force generated by the transducer to satisfy the desired dispersion equation of the Lamb wave mode.

\section{Mode Control of Lamb Waves}

\subsection{Fundamentals of Meander-Line EMAT}

Previous studies revealed that there are three coupling mechanisms that contribute to excite the guided wave in a metal plate: The Lorentz force mechanism, magnetostriction mechanism, and magnetization force mechanism, respectively. The Lorentz force mechanism arises in all conducting materials, while the magnetostriction mechanism and magnetization force mechanism appear only in ferromagnetic material [18-20]. In this work, we investigate the EMATs based on the Lorentz force mechanism to generate single-mode Lamb waves. Figure 1 shows a classical configuration of a meander-line EMAT, which consists of a permanent magnet, a coil, and a specimen. The magnet is used to provide a bias magnetic field, and the coil is used to carry the dynamic current. When a pulsed alternating current feeds to the meander-line coil, then an eddy current $(J)$ will be induced in the skin depth of the specimen. The interaction between the bias magnetic field $(B)$ and the eddy current $(J)$ produces a Lorentz force $(F)$ in the specimen, as shown in Equation (22):

$$
F=J \times B
$$

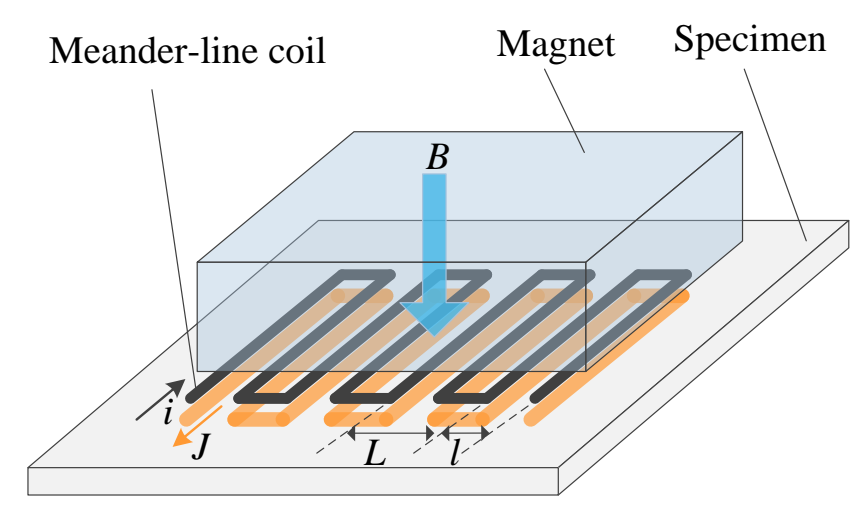

Figure 1. Schematic diagram of a meander-line coil electromagnetic acoustic transducer (EMAT).

When the particle in the skin depth vibrates with the Lorentz force, then the ultrasonic waves are generated. 
The acoustic field Equation (1) can be divided into two-dimensional wave motion equations:

$$
\begin{aligned}
& (\lambda+\mu) \frac{\partial}{\partial x}\left(\frac{\partial u}{\partial x}+\frac{\partial v}{\partial y}\right)+\mu\left(\frac{\partial^{2} u}{\partial x^{2}}+\frac{\partial^{2} u}{\partial y^{2}}\right)+F_{x}=\rho \frac{\partial^{2} u}{\partial t^{2}} \\
& (\lambda+\mu) \frac{\partial}{\partial y}\left(\frac{\partial u}{\partial x}+\frac{\partial v}{\partial y}\right)+\mu\left(\frac{\partial^{2} v}{\partial x^{2}}+\frac{\partial^{2} v}{\partial y^{2}}\right)+F_{y}=\rho \frac{\partial^{2} v}{\partial t^{2}}
\end{aligned}
$$

where $u$ and $v$ are displacements in $x$ and $y$ directions; $F_{x}$ and $F_{y}$ are Lorentz forces in $x$ and $y$ directions.

The parameters of the meander-line coil are calculated by

$$
2 L=\frac{C_{p}}{f}
$$

where $C_{p}$ is the phase velocity, $f$ is the ultrasonic frequency, and $L$ is the wavelength of the Lamb wave; the $C_{p}$ and $f$ are selected according to the dispersion curves in Figure 2. When the thickness of the specimen and the excitation frequency are given, the wavelength of the Lamb wave is determined by Equation (25). The relation between the spacing interval of adjacent wires and the wavelength is described by

$$
L=2 l(2 N+1)
$$

where $N$ is the integer $(0,1,2 \ldots \ldots)$, and $l$ is the spacing interval of adjacent wires.

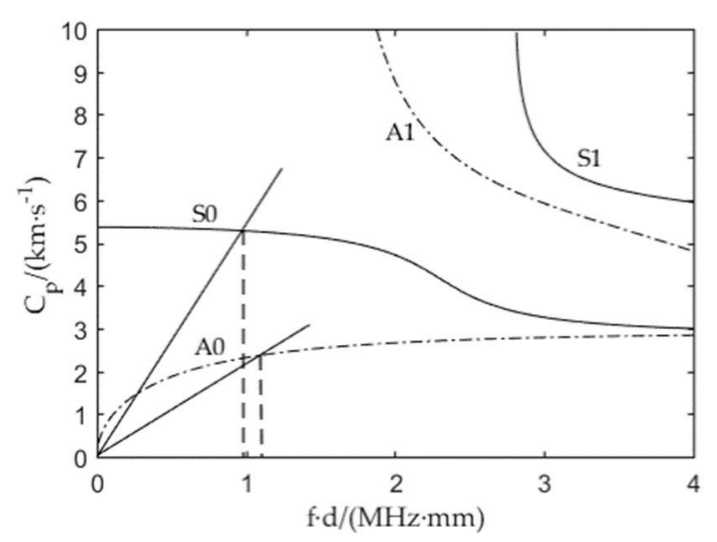

Figure 2. Phase velocity dispersion curves for A0, A1, S0, and S1 Lamb wave modes in aluminum plates.

\subsection{Modes Control with Paired EMAT Configurations}

According to the characteristic Equations (11)-(18), the displacement forms of symmetric and antisymmetric modes are shown in Figure 3, where the deformations are symmetric or antisymmetric to the middle plane of the plate, respectively. If the distributions of the excitation forces are the same as those of the displacements of the symmetric and antisymmetric motion, the corresponding Lamb wave modes will be obtained from the stress-strain relationship of elastic materials. Therefore, a paired EMAT configuration is proposed based on the mechanism of Lorentz force to generate the forces corresponding to symmetric motion. The paired EMAT consists of two traditional meander-line NB-EMATs which embrace the specimen symmetrically, as shown in Figure 4a, where the normal bias magnetic fields are provided by two vertically polarized magnets. The Lorentz forces excited by this configuration are symmetric from the middle plane of the plate, as shown in Figure $4 \mathrm{~b}$. The traction forces on the upper and lower surfaces are expressed as

$$
\tau_{y x}=\left\{\begin{array}{cc}
-F_{x} & y=d \\
-F_{x} & y=-d
\end{array} \text { and } \tau_{y y}=\left\{\begin{array}{cc}
F_{y} & y=d \\
-F_{y} & y=-d
\end{array}\right.\right.
$$




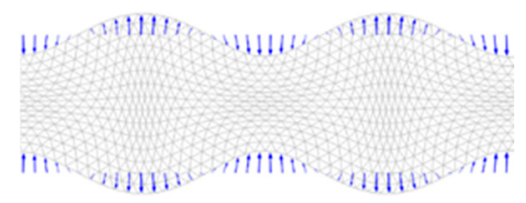

(a)

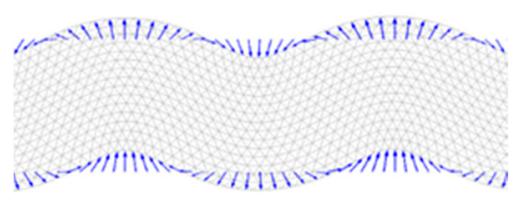

(b)

Figure 3. The displacement form of symmetric and antisymmetric Lamb waves: (a) symmetric mode, and (b) antisymmetric mode.

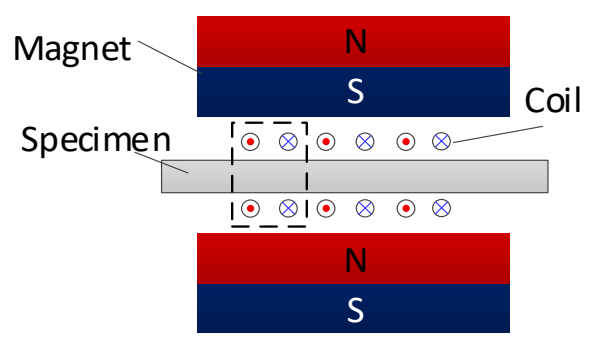

(a)

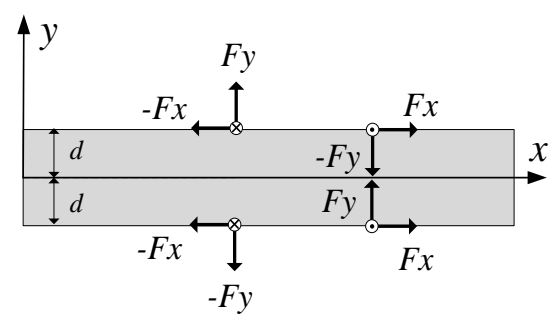

(b)

Figure 4. (a) The paired normal biased EMAT (NB-EMAT) configuration, and (b) the Lorentz forces generated by the paired NB-EMAT. Only the Lorentz forces generated by the wires in the dotted frame are plotted in (b). The symbol $\otimes$ denotes the inward current flows on the paper vertically, and $\odot$ denotes the outward current flows on the paper vertically.

Substituting the boundary loading Equation (27) into Equations (9) and (10), respectively, we can obtain

$$
\begin{aligned}
\left.\tau_{y x}\right|_{y=d}= & \mu\left[-2 i k p A_{2} \sin (p d)+\left(k^{2}-q^{2}\right) B_{1} \sin (q d)\right] \\
& +\mu\left[2 i k p A_{1} \cos (p d)+\left(k^{2}-q^{2}\right) B_{2} \cos (q d)\right]=-F_{x} \\
\left.\tau_{y x}\right|_{y=-d}= & \mu\left[2 i k p A_{2} \sin (p d)-\left(k^{2}-q^{2}\right) B_{1} \sin (q d)\right] \\
& +\mu\left[2 i k p A_{1} \cos (p d)+\left(k^{2}-q^{2}\right) B_{2} \cos (q d)\right]=-F_{x} \\
\left.\tau_{y y}\right|_{y=d}= & \mu\left[\left(k^{2}-q^{2}\right) A_{2} \cos (p d)-2 i k q B_{1} \cos (q d)\right] \\
& +\mu\left[\left(k^{2}-q^{2}\right) A_{1} \sin (p d)+2 i k q B_{2} \sin (q d)\right]=F_{y} \\
\left.\tau_{y y}\right|_{y=-d}= & \mu\left[\left(k^{2}-q^{2}\right) A_{2} \cos (p d)-2 i k q B_{1} \cos (q d)\right] \\
& -\mu\left[\left(k^{2}-q^{2}\right) A_{1} \sin (p d)+2 i k q B_{2} \sin (q d)\right]=-F_{y}
\end{aligned}
$$

If we subtract Equation (29) from Equation (28) and add Equation (30) to Equation (31), Equations (32) and (33) are obtained:

$$
\begin{gathered}
\mu\left[-2 i k p A_{2} \sin (p d)+\left(k^{2}-q^{2}\right) B_{1} \sin (q d)\right]=0 \\
\mu\left[\left(k^{2}-q^{2}\right) A_{2} \cos (p d)-2 i k q B_{1} \cos (q d)\right]=0
\end{gathered}
$$

Combining Equations (32) and (33), the symmetric mode frequency of Equation (20) is obtained.

It follows a similar way to excite antisymmetric wave modes. A paired PB-EMAT is proposed to generate antisymmetric Lamb waves. The paired PB-EMAT consists of two EMATs, whose bias magnetic fields are parallel to the plate surface. As shown in Figure 5a, the parallel bias magnetic fields are provided by two horizontally polarized magnets. In this case, the Lorentz forces are antisymmetrically distributed along the plate section, as shown in Figure $5 \mathrm{~b}$. The traction forces on the upper and lower surfaces are expressed as

$$
\tau_{y x}=\left\{\begin{array}{cc}
-F_{x} & y=d \\
F_{x} & y=-d
\end{array} \text { and } \tau_{y y}=\left\{\begin{array}{cc}
F_{y} & y=d \\
F_{y} & y=-d
\end{array}\right.\right.
$$




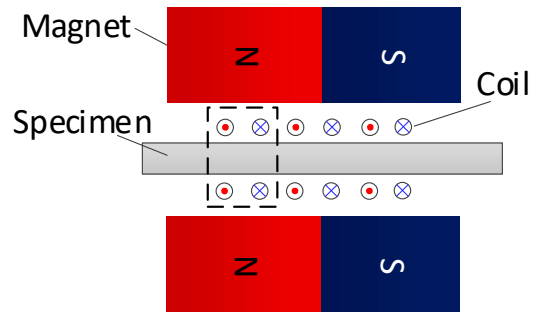

(a)

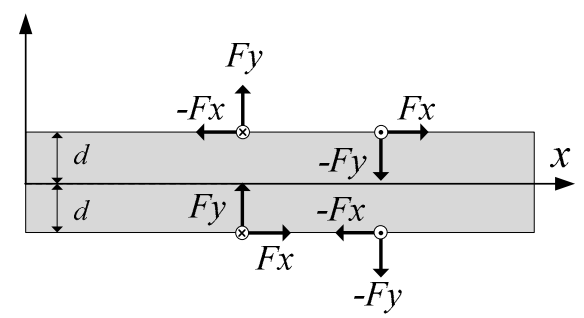

(b)

Figure 5. (a) The paired parallel biased EMAT (PB-EMAT) configuration, and (b) the Lorentz forces generated by the paired PB-EMAT. Only the Lorentz forces generated by the wires in the dotted frame are plotted in (b) too. The meanings of symbol $\bigotimes$ and $\odot$ are the same as in Figure 4.

Similarly, substituting the boundary loading Equation (34) into Equations (9) and (10), we can obtain

$$
\begin{gathered}
\mu\left[2 i k p A_{1} \cos (p d)+\left(k^{2}-q^{2}\right) B_{2} \cos (q d)\right]=0 \\
\mu\left[\left(k^{2}-q^{2}\right) A_{1} \sin (p d)+2 i k q B_{2} \sin (q d)\right]=0
\end{gathered}
$$

Combining Equations (35) and (36), the antisymmetric mode frequency Equation (21) is obtained.

Based on the above deductions, it is reasonable to infer that the paired NB-EMAT in Figure 4a can generate symmetric mode Lamb waves, whereas the structure in Figure $5 \mathrm{a}$ can generate antisymmetric mode Lamb waves. However, Figure 2 also indicates that the Lamb waves have multimode characteristics, and the number of Lamb wave modes increases with the increase of frequency-thickness product. Therefore, the appropriate cut-off frequency should be selected to ensure that only one mode is generated. As the dashed line shown in Figure 2 demonstrates, only the $\mathrm{S} 0$ and A0 modes exist when the frequency-thickness product is below $1.6 \mathrm{MHz} \cdot \mathrm{mm}$. In this paper, the frequency-thickness product $0.984 \mathrm{MHz} \cdot \mathrm{mm}$ and $1.1 \mathrm{MHz} \cdot \mathrm{mm}$ are used for $\mathrm{S} 0$ and A0, respectively.

\section{Verifications}

\subsection{Simulation for Modes Control of Lamb Waves}

In this section, the $\mathrm{S} 0$ and $\mathrm{A} 0$ modes controlled by using paired EMATs are verified by simulations and experiments.

Firstly, the modes selectivity of conventional structure EMATs and paired structure EMATs are compared, and the EMAT models are set up in a commercial finite element analysis software, COMSOL Multiphysics. The electromagnetic field and the mechanics field were used. The 'AC/DC, Magnetic Fields' module is used to generate static bias magnetic and induced eddy current in the specimen. The 'Solid Mechanics (Elastic wave)' module is used to calculate body loads that are the Lorentz forces generated in the skin depth of the specimen. The parameters for symmetric and antisymmetric EMATs are selected and calculated in Table 1. A narrow bandwidth 5-peak tone burst signal is used as excitation source, because the dispersion of a Lamb wave is more obvious with the increase of bandwidth [21]. An observation point was set on the surface of the specimen to detect the wave motion in the plate. The distance between the observation point and the EMATs is $400 \mathrm{~mm}$.

Table 1. The parameters of EMATs.

\begin{tabular}{ccccccc}
\hline Mode & Phase Velocity (m/s) & $\begin{array}{c}\text { Frequency Thickness } \\
\text { Product (MHz } \mathbf{m m})\end{array}$ & Center Frequency (kHz) & Magnet (mm) & Wavelength (mm) & Plate Thickness (mm) \\
\hline S0 & 5262 & 0.984 & 328 & $57 \times 37 \times 17$ & 16 & 3 \\
A0 & 2442 & 1.1 & 366.7 & $37 \times 37 \times 37$ & 6.3 & 3 \\
\hline
\end{tabular}


Figure 6 shows the simulation results, where the amplitude ratio of the $\mathrm{S} 0$ to $\mathrm{A} 0$ is $16.8 \mathrm{~dB}$ for the conventional symmetric mode EMAT (shown in Figure 6a), and $50.2 \mathrm{~dB}$ for the paired NB-EMAT (shown in Figure $6 \mathrm{~b}$ ). The amplitude ratio of the $\mathrm{A} 0$ to $\mathrm{S} 0$ is $21 \mathrm{~dB}$ for the conventional antisymmetric mode EMAT (shown in Figure 6c), and $83.8 \mathrm{~dB}$ for the paired PB-EMAT (shown in Figure 6d). Therefore, we can find that the paired structure EMATs have better mode selectivity compared to the conventional EMATs.

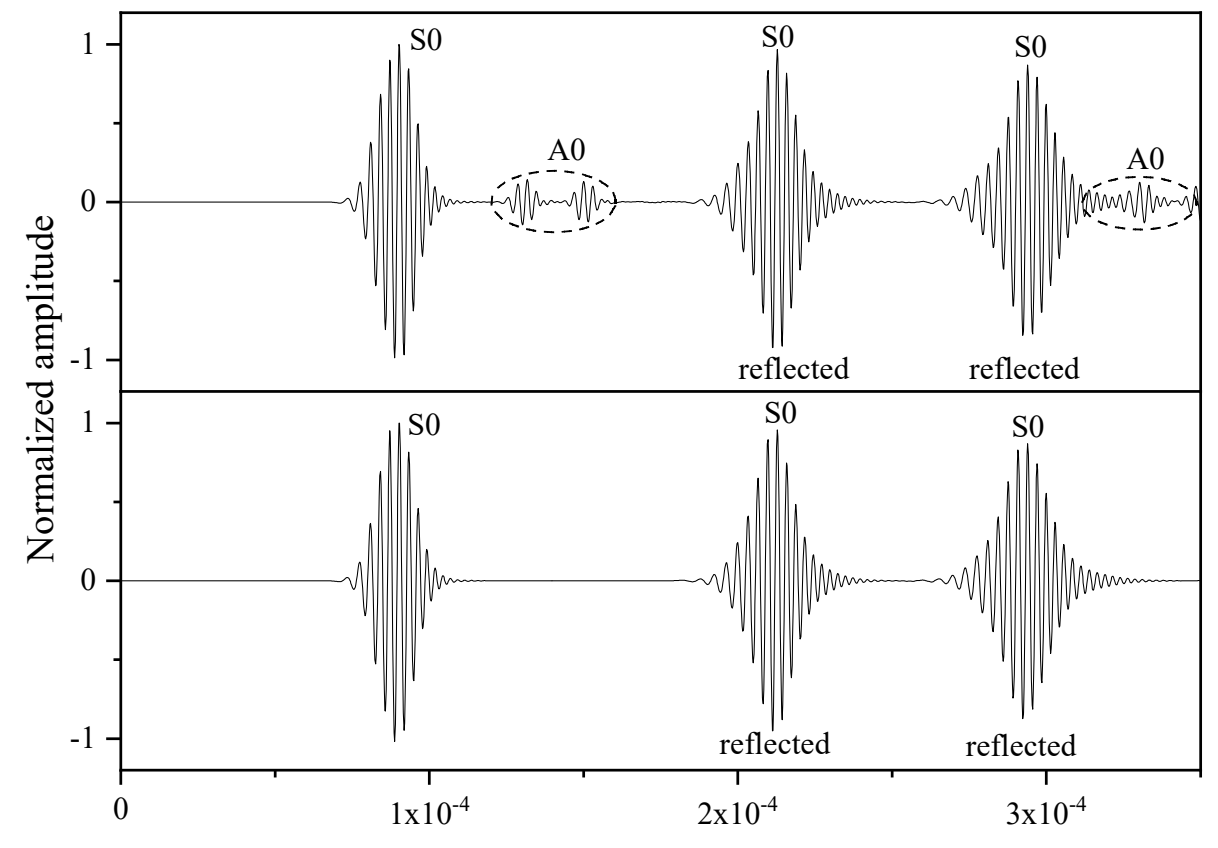

(a)

Time (s)

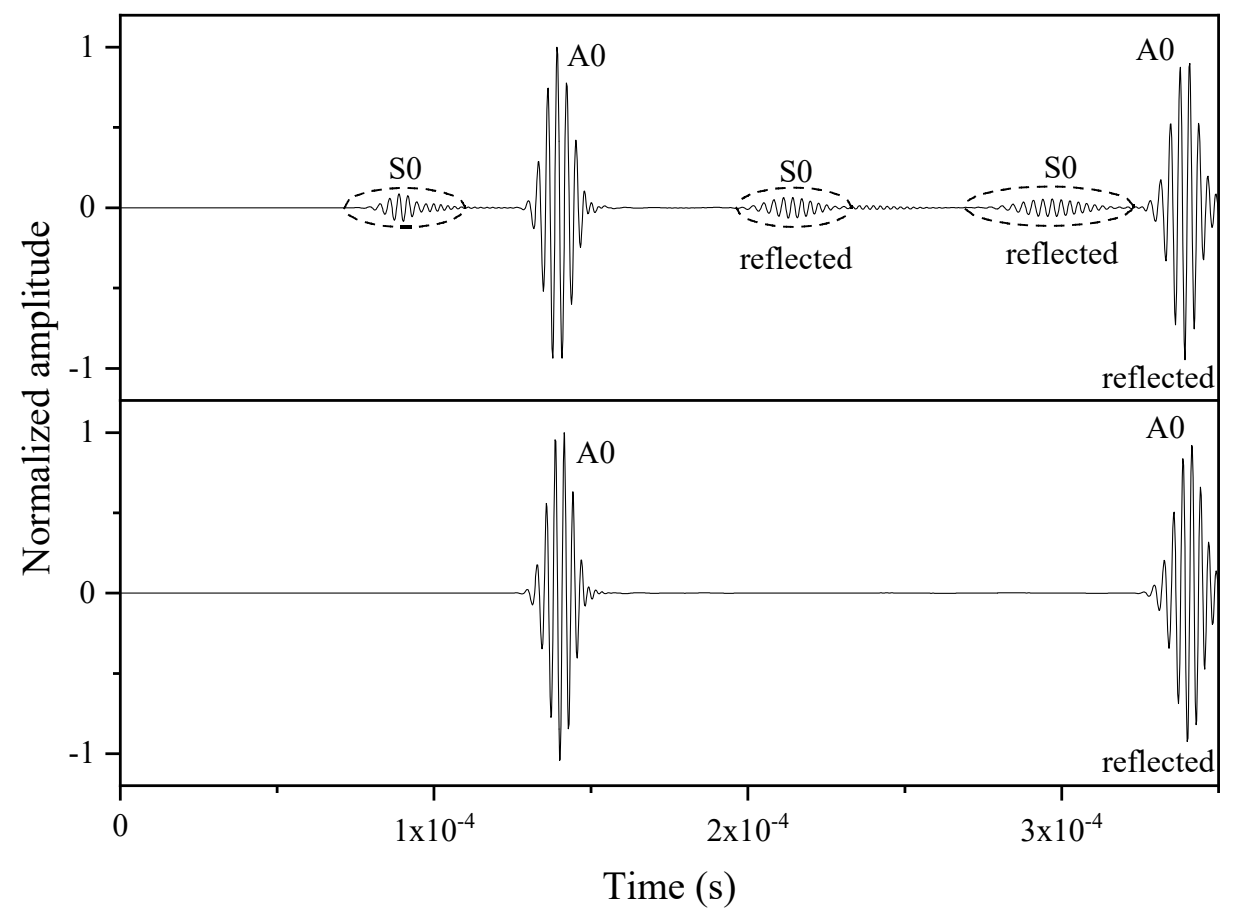

(c)

(d)

Figure 6. Results of simulation. The observed Lamb wave harmonic displacement, $\mathrm{S} 0$ mode, generated by (a) traditional EMAT and (b) paired NB-EMAT; A0 mode, generated by (c) traditional EMAT and (d) paired PB-EMAT. 


\subsection{Experimental Setup}

Next, the modes controlled by using paired structure EMATs are validated by various experiments (as shown in Figure 7), where a paired structure EMAT consists of two traditional EMATs. The parameters of the coils and magnets used in the experiments are the same as those used in simulations, which are given in Table 1. The coils are fabricated by a printed circuit board (PCB). The upper coil and lower coil are series connected. Figure 8 represents the photograph of the transmitter, where two cross slides are installed on the shelves, which are made of aluminum alloy profiles. Each slide can be moved both in the $X$ and $Z$ direction by turning the handwheels. Both the lower EMAT and upper EMAT are fixed on the cross slides. The magnets and coils are installed on a seat. The position and lift-off height of EMATs can be adjusted by turning the handwheel, which is installed on the terminal of the lead screws. The dial scale on the handwheel is used to precisely locate the EMATs' positions. Accurate alignment of upper and lower EMATs can be achieved by adjusting the positions of $\mathrm{X}$ and $\mathrm{Z}$ handwheels.

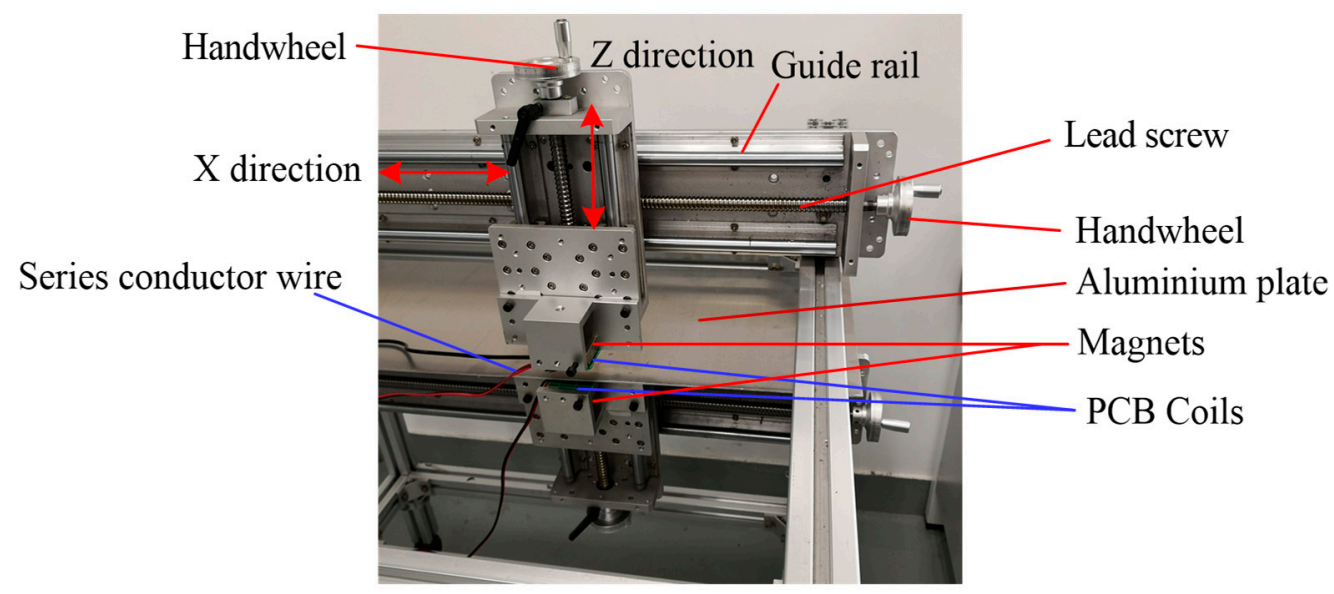

Figure 7. Experimental setup. The upper EMAT and lower EMAT are installed on the cross slides symmetrically, and printed circuit board (PCB) coils are series connected by conductor wire.

A function signal generator is used to generate a narrow bandwidth 5-peak signal, which is a cosine signal modulated by the Hanning window. An impedance matching circuit is connected between the transmitting EMAT and pulse power amplifier to enhance the efficiency of power conversion. The receiver is connected to both a filter and a programmable amplifier, which are integrated in the power amplifier. The output signals are displayed on the digital storage oscilloscope. The transmitter and the receiver are separated with a distance of $400 \mathrm{~mm}$ between them. The receiver is instead by a traditional EMAT.

Figure 8 shows the experimental results, where Figure $8 \mathrm{a}, \mathrm{c}$ are the ultrasonic signals generated by the traditional symmetric and antisymmetric EMATs. The amplitude ratio of the $\mathrm{S} 0$ to $\mathrm{A} 0$ is $11.8 \mathrm{~dB}$ for the conventional symmetric mode EMAT. The amplitude ratio of the A0 to $\mathrm{S} 0$ is $25.7 \mathrm{~dB}$ for the conventional antisymmetric mode EMAT. Figure $8 \mathrm{~b}$ shows the $\mathrm{S} 0$ mode ultrasonic signals generated by the paired structure EMATs, and the ratio of $\mathrm{S} 0$ mode to A0 mode is $36 \mathrm{~dB}$. Similarly, in Figure 8d, the ratio of $\mathrm{A} 0$ mode to $\mathrm{S} 0$ mode is $36.9 \mathrm{~dB}$. However, the signal to noise ratio (SNR) is about $36 \mathrm{~dB}$ in the experiment. In other words, the amplitudes of unwanted modes are as same as the noise.

The results of simulations and experiments verify that the paired structure EMATs can control the generation of single $\mathrm{S} 0$ and $\mathrm{A} 0$ mode Lamb waves. These EMATs will help improve the application of Lamb waves in nondestructive testing and evaluation. 


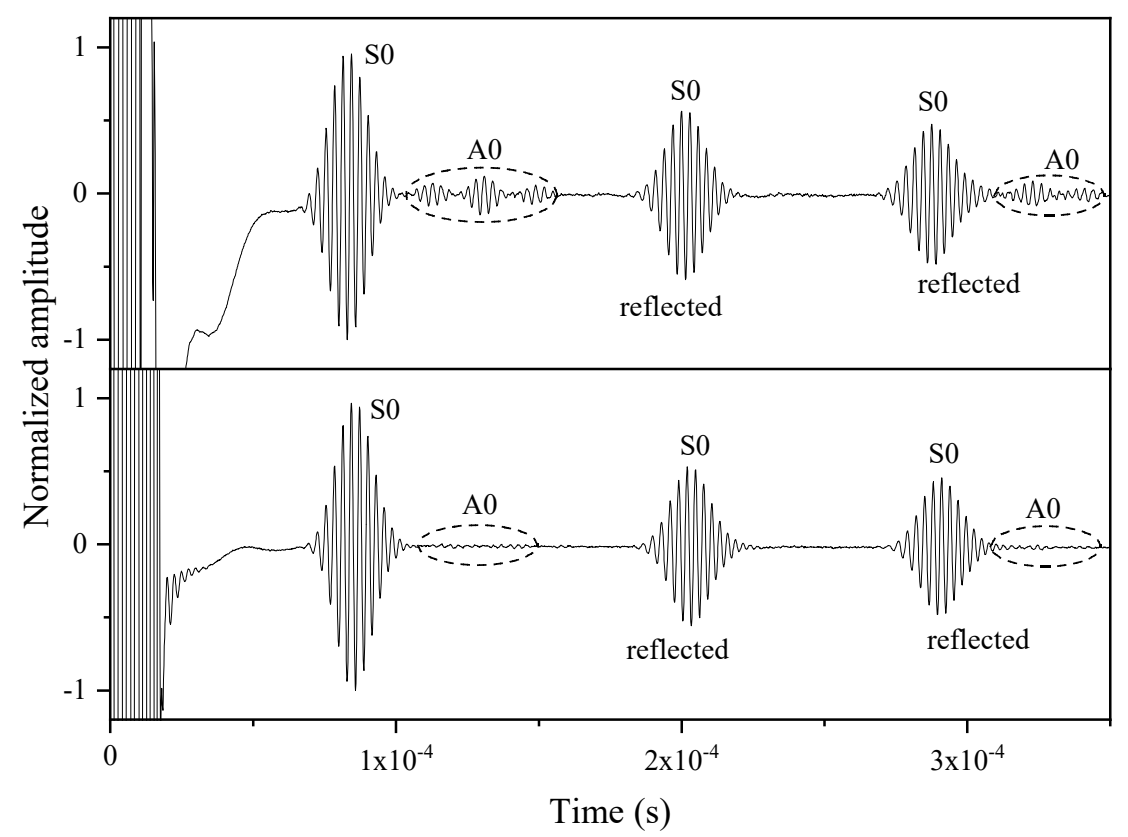

(a)

(b)

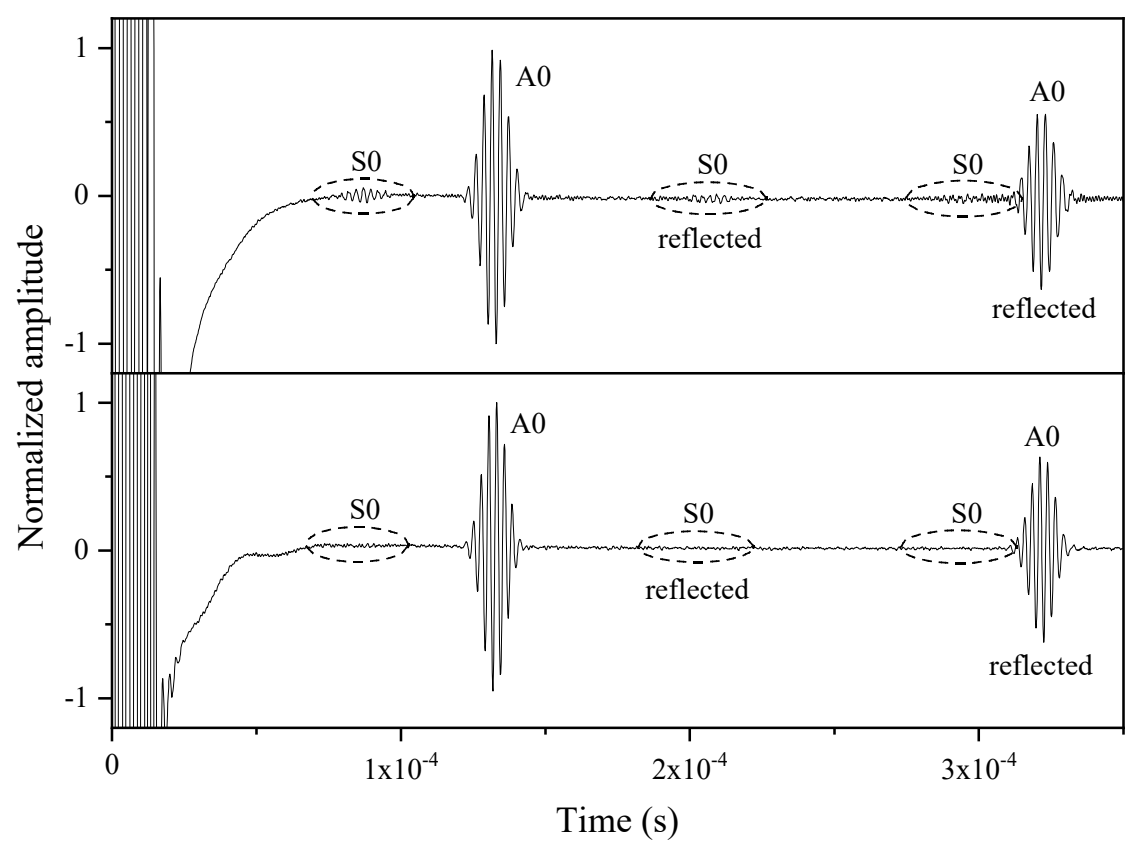

(c)

(d)

Figure 8. Results of the experiment. The collected Lamb wave signals, S0 mode, generated by (a) traditional EMAT and (b) paired NB-EMAT; A0 mode, generated by (c) traditional EMAT and (d) paired PB-EMAT.

\section{Conclusions}

Multimodality is one of the main characteristics of guided waves. The generation of single-mode Lamb waves by the meander-line coil EMAT is always a difficult problem. This paper described the design of paired structure EMATs for aluminum plate-like structures. Following theoretical considerations, the configurations to excite single guided wave modes are discussed. Two types of transducer designs are studied, namely the paired NB-EMAT and PB-EMAT. Using two-dimensional finite element modeling, it was shown that the surface loading force of the plates differs from one 
another, leading to different displacement patterns on a structure. In the paired NB-EMAT design, the loading force on the structure is symmetrical to the midplane of the plate. In the paired PB-EMAT design, the loading force on the structure is antisymmetrical to the midplane of the plate.

By comparing the signal of Lamb wave excited by the traditional EMATs with that by the proposed paired configuration EMATs through simulation and experiment, it can be determined that the excitation force has a greater influence on the motion form of the guided wave. When the excitation force is symmetrical with the midplane of the plate, the symmetrical mode Lamb wave is excited, and the antisymmetrical excitation force excites antisymmetrical Lamb waves. If the excitation force only acts on one side of the plate, the force distribution cannot make the wave motion satisfy the single-mode dispersion equation, so the $\mathrm{S} 0$ mode and $\mathrm{A} 0$ mode will be generated at the same time. Therefore, to excite a single-mode guided wave in the true sense, it is very important that the excitation force meets the boundary conditions of the single-mode dispersion equation, as is the case in the design of other types of transducers.

Author Contributions: conceptualization, validation, and writing-original draft preparation, Y.Z.; resources, project administration, and funding acquisition, Z.Q.; methodology, and writing-review and editing, B.W. All authors have read and agreed to the published version of the manuscript.

Acknowledgments: This work was funded by the National Natural Science Foundation of China (No. 11611530686), the State Key Laboratory of Mechanics and Control of Mechanical Structures at NUAA [MCMS-E-0520K02], and the Key Laboratory of Impact and Safety Engineering, Ministry of Education at Ningbo University [CJ201904], the Guangxi Key Laboratory of Manufacturing System and Advanced Manufacturing Technology (Grant Nos. 17-259-05-005Z, 17-259-05-007Z), and the Priority Academic Program Development of Jiangsu Higher Education Institutions (PAPD).

Conflicts of Interest: The authors declare no conflict of interest.

\section{References}

1. Dalton, R.P.; Cawley, P.; Lowe, M.J.S. The Potential of Guided Waves for Monitoring Large Areas of Metallic Aircraft Fuselage Structure. J. Nondestruct. Eval. 2001, 20, 29-46. [CrossRef]

2. Huang, S.L.; Wei, Z.; Zhao, W.; Wang, S. A New Omni-Directional EMAT for Ultrasonic Lamb Wave Tomography Imaging of Metallic Plate Defects. Sensors 2014, 14, 3458-3476. [CrossRef] [PubMed]

3. Kojima, F.; Furusawa, A.; Ito, T. Impact Model and Control of Ultrasonic Excitation using Electromagnetic Acoustic Transducer. In Proceedings of the 2015 10th Asian Control Conference (Ascc), Sabah, Malaysia, 31 May-3 June 2015; 2015.

4. Su, Z.; Ye, L.; Lu, Y. Guided Lamb waves for identification of damage in composite structures: A review. J. Sound Vib. 2006, 295, 753-780. [CrossRef]

5. Li, D.; Zhang, S.; Yang, W.; Zhang, W. Corrosion Monitoring and Evaluation of Reinforced Concrete Structures Utilizing the Ultrasonic Guided Wave Technique. Int. J. Distrib. Sens. Netw. 2014, 10, 827130. [CrossRef]

6. Shen, W.; Li, D.; Zhang, S.; Ou, J. Analysis of wave motion in one-dimensional structures through fast-Fourier-transform-based wavelet finite element method. J. Sound Vib. 2017, 400, 369-386. [CrossRef]

7. Su, Z.; Ye, L. Selective generation of Lamb wave modes and their propagation characteristics in defective composite laminates. J. Mater. Des. Appl. 2004, 218, 95-110. [CrossRef]

8. Koduru, J.P.; Rose, J.L. Transducer arrays for omnidirectional guided wave mode control in plate like structures. Smart Mater. Struct. 2013, 22, 015010. [CrossRef]

9. Glushkov, E.V.; Glushkova, N.V.; Kvasha, O.V.; Lammering, R. Selective Lamb mode excitation by piezoelectric coaxial ring actuators. Smart Mater. Struct. 2010, 19, 035018. [CrossRef]

10. Giurgiutiu, V. Tuned Lamb Wave Excitation and Detection with Piezoelectric Wafer Active Sensors for Structural Health Monitoring. J. Intell. Mater. Syst. Struct. 2016, 16, 291-305. [CrossRef]

11. Seher, M.; Huthwaite, P.; Lowe, M.J.S.; Nagy, P.B. Model-Based Design of Low Frequency Lamb Wave EMATs for Mode Selectivity. J. Nondestruct. Eval. 2015, 34. [CrossRef]

12. Lee, J.K.; Kim, Y.Y. Tuned double-coil EMATs for omnidirectional symmetric mode lamb wave generation. NDT E Int. 2016, 83, 38-47. [CrossRef]

13. Sun, W.; Liu, G.; Xia, H.; Xia, Z. A modified design of the omnidirectional EMAT for antisymmetric Lamb wave generation. Sens. Actuators A Phys. 2018, 282, 251-258. [CrossRef] 
14. Liu, Z.; Hu, Y.; Xie, M.; Wu, B.; He, C. Development of omnidirectional A 0 mode EMAT employing a concentric permanent magnet pairs with opposite polarity for plate inspection. NDT E Int. 2018, 94, 13-21. [CrossRef]

15. Graff, K.F. Wave Motion in Elastic Solids; Courier Corporation: Oxford, UK; p. 1975.

16. Achenbach, J.D. Wave Propagation in Solids; North-Holland Publishing Company: Amsterdam, The Netherlands, 1999.

17. Rose, J.L. Ultrasonic Waves in Solid Media; Cambridge University Press: Cambridge, UK, 1999.

18. Kaltenbacher, M.; Ettinger, K.; Lerch, R. Finite element analysis of coupled electromagnetic acoustic systems. IEEE Trans. Magn. 1999, 35, 1610-1613. [CrossRef]

19. Murayama, R. Driving mechanism on magnetostrictive type electromagnetic acoustic transducer for symmetrical vertical mode Lamb wave and for shear horizontal mode plate wave. Ultrasonics 1996, 34, 729-736. [CrossRef]

20. Ashigwuike, E.C.; Ushie, O.J.; Mackay, R.; Balachandran, W. A study of the transduction mechanisms of electromagnetic acoustic transducers (EMATs) on pipe steel materials. Sens. Actuators A Phys. 2015, 229, 154-165. [CrossRef]

21. Wilcox, P.D.; Dalton, R.P.; Lowe, M.J.S.; Cawley, P. Mode and Transducer Selection for Long Range Lamb Wave Inspection. Key Eng. Mater. 1999, 167-168, 152-161. [CrossRef]

(C) 2020 by the authors. Licensee MDPI, Basel, Switzerland. This article is an open access article distributed under the terms and conditions of the Creative Commons Attribution (CC BY) license (http://creativecommons.org/licenses/by/4.0/). 